\title{
Phasic abnormalities of left ventricular emptying in coronary artery disease
}

\author{
S WALTON, J YIANNIKAS, P H JARRITT, N J G BROWN, \\ R H SWANTON, P J ELL
}

\begin{abstract}
From the Department of Cardiology and Institute of Nuclear Medicine, Middlesex Hospital and Medical School, London
\end{abstract}

SUMMARY Seventy subjects with suspected coronary artery disease were studied by radionuclide angiocardiography. Delayed or paradoxically emptying regions of the left ventricle were detected by a relatively new nuclear technique-phase imaging. The results were assessed in the light of cardiac catheterisation findings. Compared with 19 normals, regions with abnormally high phase (and therefore late emptying) were found in 42 of 61 subjects with coronary disease. High phase values were associated with total occlusion of a major coronary artery, low ejection fraction, and extensive wall motion abnormalities. The phase image greatly facilitated the calculation of contractile segment ejection fraction in 14 cases of left ventricular aneurysm. In three of these postoperative left ventricular ejection fraction agreed closely with preoperative contractile segment ejection fraction and there was a distinct improvement in the phase image after aneurysmectomy.

Radionuclide angiocardiography can provide accurate estimates of ventricular ejection fraction but problems with edge definition make wall motion studies less reliable. The purpose of this study is to evaluate a nuclear technique which can detect regional ventricular abnormalities but which does not involve wall motion assessment. The phase image is an image of the intracardiac blood pool. It is produced by analysis of activity time curves from individual display elements during blood pool scintigraphy. Display elements within the cardiac outline show phasic fluctuations in activity with each heart beat, the timing of these fluctuations being different in different chambers. To produce the phase image each display element is colour coded according to the timing of these fluctuations relative to the $R$ wave of the electrocardiogram. Thus, regions that are in phase are shown in the same colour. Regions that are out of phase are shown in different colours. Not only are atria distinguished from ventricles but also within the ventricles regions with delayed or paradoxical emptying are identified.

\section{Subjects and methods}

Seventy subjects with suspected coronary artery disease were studied by radionuclide angiocardiography. Ages ranged between 28 and 74 (mean

Received for publication 20 January 1981
53). Sixty-one were men. Sixty also underwent cardiac catheterisation.

\section{RADIONUCLIDE STUDIES}

In vivo erythrocyte labelling was achieved by sequential intravenous administration of $5 \mathrm{mg}$ stannous pyrophosphate and $20 \mathrm{mCi}^{99}$ technetium pertechnetate. After equilibration a General Electric IGE $400 \mathrm{~T}$ wide field of view gamma camera, fitted with a high sensitivity parallel hole collimator and positioned in the left anterior oblique projection was used to accumulate 2 million counts per study. Electrocardiographic gating was used to identify individual cardiac cycles. Data were stored on disc and analysed using the standard software of an Informatek Simis 3 computer system. A 16 frame representative cine cycle was produced and used as the basis for further analysis. Thus for each display element a 16 point activity time curve was produced by plotting activity for that element for each image in the representative cycle. For each of these curves phase was calculated, being defined as the angle whose tangent is given by the ratio of the amplitudes of the sine and cosine waves of the fundamental frequency of the Fourier transform. ${ }^{1}$ To produce the phase image each display element was colour coded according to its phase using a 64 element colour scale. Four regions were routinely outlined on the phase image- the whole left ventricle and three segments of it, lateral, septal, and inferior (as shown in Fig. 1). 
Mean phase was calculated for each segment. Using those subjects who turned out to be normal, the normal phase values were established for each segment. Subsequently, values differing from the mean by more than two standard deviations were considered abnormal.

Left ventricular ejection fraction was calculated from activity changes within the left ventricular region of interest. In some subjects with segments showing a very high phase, and therefore suggesting left ventricular aneurysm, ejection fraction was also calculated from a region of interest over the left ventricle but excluding the high phase region-the contractile segment ejection fraction.

Fifteen subjects underwent consecutive radionuclide studies at rest to establish the reproducibility of the technique. They were a heterogeneous group with a wide range of ejection fraction values ( 10 to 51 , mean $38 \%$ ). Three subjects had studies before and within one month after left ventricular aneurysmectomy.

\section{CARDIAC CATHETERISATION}

Coronary arteriography was performed in at least three views by either the Sones or Judkins techniques. Left ventricular angiography was performed in the $30^{\circ}$ right anterior oblique projection. Angiograms were analysed subjectively and by superimposition of end-systolic and end-diastolic outlines. Wall motion was classified as normal, local hypokinesis or akinesis, extensive akinesis, or aneurysm.

Largely on the basis of catheterisation findings the subjects were divided into three groups

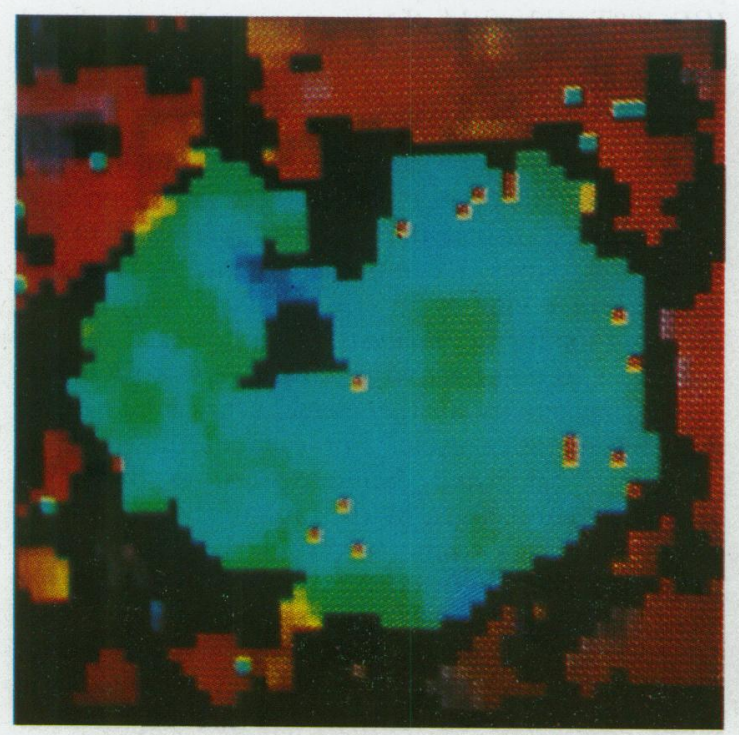

\section{Group 1}

Either Suspected coronary disease but normal coronary arteries $(n=9)$

Or Suspected coronary disease but normal exercise electrocardiogram and nuclear ejection fraction response to stress who were not subjected to catheterisation $(\mathrm{n}=10)$

\section{Group 2}

Coronary disease but without total occlusion of a major coronary artery $(n=16)$

\section{Group 3}

At least one major coronary artery totally occluded $(\mathrm{n}=35)$

The results of phase analysis were assessed in the light of catheterisation findings.

\section{Results}

Background corrected, end-diastolic left ventricular counts were between 9000 and 31000 (mean 16500 ).

Fig. 1 shows a normal phase image and Fig. 2 one from a subject with total occlusion of the left anterior descending coronary artery. In both, the ventricles are clearly distinguished from the atria. In Fig. 2, however, there is a progressive increase in phase across the left ventricle from laterally to medially, with some septal regions being in phase with the atria.

Table 1 shows mean segmental phase values and projected normal limits from the group 1 subjects. Group 1 ejection fractions were between 40 and 59

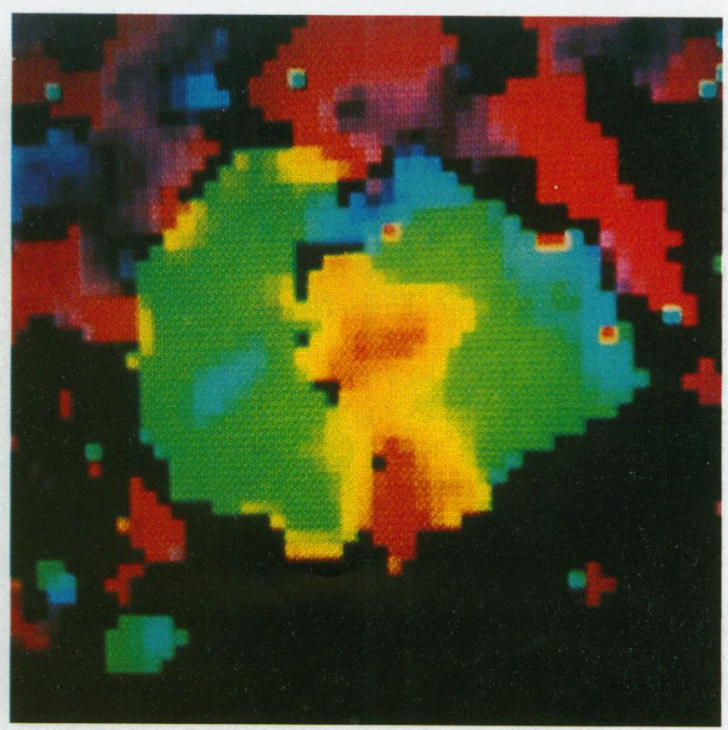

Fig. 2 
Table 1 Phase values and normal limits for normal subjects from group 1

\begin{tabular}{lll}
\hline & Mean phase & $\begin{array}{l}\text { Normal } \\
\text { upper limit }\end{array}$ \\
\hline \multirow{4}{*}{ Region } & &
\end{tabular}

Table 2 Phase, ejection fraction, and angiographic results for group 2

\begin{tabular}{|c|c|c|c|c|c|}
\hline \multirow[t]{2}{*}{ No. } & \multicolumn{3}{|c|}{ Abnormal phase } & \multirow[t]{2}{*}{$L V E F$} & \multirow[t]{2}{*}{ Angiogram } \\
\hline & $L$ & $S$ & $I$ & & \\
\hline 20 & & 200 & 181 & 34 & Localised akinesis \\
\hline 21 & & 185 & 199 & 45 & Normal \\
\hline 22 & & & & 36 & Normal \\
\hline 23 & & & & 47 & Localised hypokinesis \\
\hline 24 & & & & 42 & Normal \\
\hline 25 & & & & 54 & Localised hypokinesis \\
\hline 26 & & & & 37 & Normal \\
\hline 27 & & & & 57 & Normal \\
\hline 28 & & & & 43 & Normal \\
\hline 29 & & & & 54 & Normal \\
\hline 30 & & & 209 & 28 & Localised akinesis \\
\hline 31 & 179 & 182 & 197 & 68 & Normal \\
\hline 32 & & & & 47 & Normal \\
\hline 33 & & & & 52 & Normal \\
\hline 34 & 182 & 186 & 209 & 40 & Localised akinesis \\
\hline 35 & & 186 & & 52 & Localised hypokinesis \\
\hline
\end{tabular}

L, lateral; S, septal; I, inferior regions; LVEF, left ventricular ejection fraction. Normal phase values not shown. (mean 51)\%. All nine subjects who underwent catheterisation had normal ventriculograms.

Phase, ejection fraction, and ventriculographic results for the group 2 and 3 subjects are summarised in Table 2 and 3, respectively. Eight group 2 subjects had either a low ejection fraction or an abnormal ventriculogram or both. In group 3 there was a wide range of ejection fraction values andiventriculographic findings.

Considering groups 2 and 3 together, 19 subjects had normal radiographic ventriculograms. Six of these had high phase values (178 to 199 , mean $189^{\circ}$ ). The ejection fractions of the 19 were between 36 and 68 (mean 48)\%.

Eight subjects had localised hypokinesis and three of these had abnormal phase values $\left(186^{\circ}, 183^{\circ}\right.$, and $202^{\circ}$ ). Ejection fractions of the eight ranged between 37 and 59 (mean 48)\%.

Of 10 subjects with localised akinesis, none had a normal phase image. Nine had abnormal mean segmental phase values $\left(179\right.$ to 235 , mean $\left.202^{\circ}\right)$. One subject had a region of high phase which was too small to affect mean segmental values. Ejection fractions were between 17 and 40 (mean 31)\%.

Fourteen subjects had extensive areas of akinesis or aneurysms. All had at least one segment of abnormally high phase (193 to 280 , mean $221^{\circ}$ ). Ejection fractions were between 10 and 36 (mean 22)\%.

Pre- and postoperative results in the three subjects who underwent aneurysmectomy are shown in Table 4.

In the 15 subjects who had two nuclear studies the

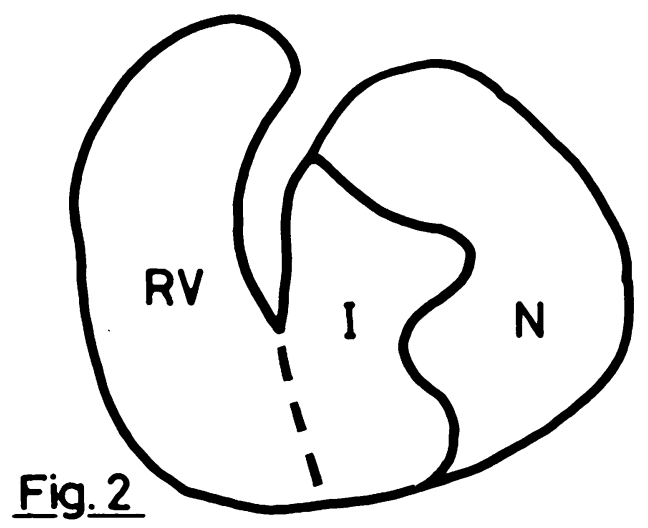

Fig. 1 Normal phase image. $R V$, right ventricle; $S, I, L$ septal, inferior, and lateral regions of the left ventricle. The colour scale is such that low phase values are shown as blue. Progressive increase in phase is represented by green, yellow, red, and purple.

Fig. 2 Phase image from a subject with an anterior myocardial infarction, $R V$, right ventricle; $N$, normally emptying region of the left ventricle; $I$, infarcted region. Note the progressive delay in emptying across the left ventricle from lateral to medial. 
Table 3 Phase, ejection fraction, and angiographic results for group 3

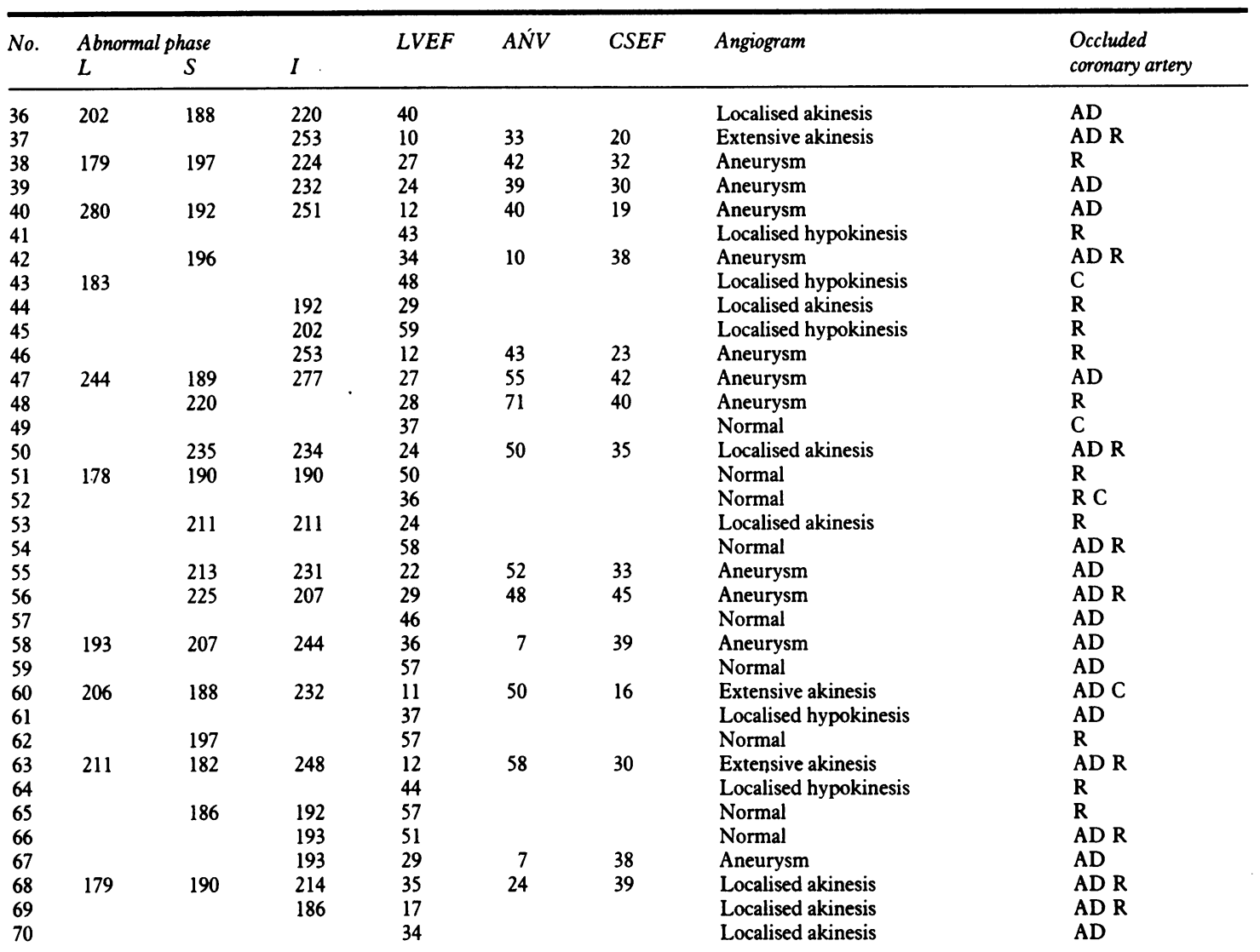

L, lateral; S, septal; I, inferior segments; LVEF, left ventricular ejection fraction; CSEF, contractile segment ejection fraction; AD, anterior descending; $R$, right; $C$, circumflex coronary arteries; ANV, aneurysmal volume (\% LV volume). Normal values not shown.

difference between estimated segmental phase values was $6.0 \pm 4.4 \%$ (mean $\pm S D)$.

\section{Discussion}

Endocardial activation times differ by up to $20 \mathrm{~ms}$, the apex in particular showing late activation. ${ }^{2}$ Initiation of contraction varies by up to $35 \mathrm{~ms}$, the apex being the last region to contract. ${ }^{3}$ These findings probably explain consistently higher phase values in the normals in the inferior segment which contains the apex. Regional differences were taken into account by establishing a normal range for each segment.

This study compares phase analysis with the extent of coronary disease, left ventricular ejection fraction, and contrast ventriculography. Of these the last

Table 4 Pre-and postoperative findings in three subjects who underwent left ventricular aneurysmectomy

\begin{tabular}{|c|c|c|c|c|c|c|c|c|c|}
\hline \multirow[t]{3}{*}{ No. } & \multicolumn{3}{|c|}{ Preoperative } & \multirow{3}{*}{ LVEF } & \multirow{3}{*}{ CSEF } & \multicolumn{3}{|c|}{ Postoperative } & \multirow{3}{*}{$L V E F$} \\
\hline & & rmal & & & & & rmal & & \\
\hline & $L$ & $S$ & $I$ & & & $L$ & $S$ & $I$ & \\
\hline 42 & & 196 & & 34 & 38 & & & & 37 \\
\hline 47 & 244 & 189 & 277 & 27 & 42 & 225 & 210 & 218 & 40 \\
\hline 48 & & 220 & & 28 & 40 & & & & 39 \\
\hline
\end{tabular}

LVEF, left ventricular ejection fraction; CSEF, contractile segment ejection fraction; $L$, lateral; S, septal; I, inferior segments. 
causes most problems. The amplitude of wall movement and the timing of emptying are independent variables (conduction abnormalities are the prime example). Subjective analysis of contrast ventriculograms is less reproducible than objective methods ${ }^{4}$ but fixed external reference systems produce inaccurate results because of cardiac movement. Different projections were used for the radiographic and nuclear studies. For these reasons we have not attempted to carry out a detailed comparison between the two. We analysed ventriculograms subjectively to correct for any obvious errors produced by cardiac movement when end-systolic and end-diastolic outlines were superimposed. This approach is far from ideal but it was adequate for our purpose which was, broadly, to classify ventricles into groups with increasing abnormalities. That this was achieved is reflected in the fact that mean ejection fractions for each wall motion category vary inversely with the degree of abnormality.

Not surprisingly, as any resting ventriculographic technique has limited sensitivity in the detection of coronary disease, several group 2 and 3 subjects had normal phase images. These normal findings were largely supported by normal or near normal ejection fractions (35 to 57 , mean $45 \%$ ) and ventriculograms (10 normal, four local hypokinesis). Two group 2 subjects (21 and 31 ) had abnormal phase images despite normal ejection fractions and ventriculograms. This may reflect the poor sensitivity of the latter two techniques ${ }^{56}$ or it may be that phase is affected by loading conditions as one subject was hypertensive and the other had a raised left ventricular end-diastolic pressure. In three group 2 subjects abnormal phase values were supported by low ejection fractions and abnormal ventriculograms.

The higher phase values were only found in association with total occlusion of a major coronary artery. It was not possible reliably to predict the site of coronary occlusion from the phase images (Table 5) because of the superimposition of the territories of different coronary arteries in the left anterior oblique projection.

Ejection fraction values obtained using the nuclear technique are somewhat lower than values we would expect to obtain from a radiographic approach (40 to $59 \%$ ). Once having defined a normal range, however, phase imaging was not superior to ejection fraction estimation in the differentiation between normal and abnormal ventricles. It was superior, however, in the distinction between regional and global ventricular abnormalities.

Perhaps the greatest advantage of phase imaging is that it can quantify the degree of abnormality. Within a particular ventricle there is often a continuous
Table 5 Comparison of segents with abnormal phase with site of coronary occlussion

\begin{tabular}{|c|c|c|c|c|}
\hline & \multicolumn{4}{|c|}{ Abnormal region ( 3 regions per ventricle) } \\
\hline & None & Lateral & Septal & Inferior \\
\hline AD (11) & 4 & 4 & 5 & 6 \\
\hline R (11) & 3 & 2 & 6 & 7 \\
\hline C (2) & 1 & 1 & & \\
\hline$A D$ and $C$ & & 1 & 1 & 1 \\
\hline $\mathrm{R}$ and $\mathrm{C}$ (1) & 1 & & & \\
\hline $\mathrm{AD}$ and $\mathrm{R}$ & 1 & 2 & 5 & 7 \\
\hline
\end{tabular}

$\mathrm{AD}$, anterior descending; $\mathrm{R}$, right; $\mathrm{C}$, circumflex coronary arteries. Numbers in parentheses are totals of subjects with the particular combination of coronary artery occlusion stated.

distribution of phase. Thus, in functional terms, the distinction between aneurysms, regions with delayed emptying, and normal regions is a quantitative rather than a qualitative matter. It is merely a matter of degree.

Of the 14 subjects with extensive akinetic or aneurysmal regions on the ventriculogram, only two did not have a mean segmental phase value in excess of $220^{\circ}$. Even in these two, high phase regions within the ventricle were obvious but they were too small to affect the mean segmental value. Conversely, phase values above $220^{\circ}$ were confined to the extensive akinesis or aneurysm group with one exception-a subject with a localised area of akinesis and an ejection fraction of $24 \%$. In some subjects very high phase values of 250 to $280^{\circ}$ were found. Again, these figures being mean segmental values underestimate peak phase.

Traditionally, the clinical diagnosis and assessment of ventricular aneurysm have been made from the ventriculogram. The distinction between paradoxical and normally contracting segments is, however, by no means straightforward. The degree of paradoxical wall motion is usually small and, even on good quality angiograms, there is a certain amount of inaccuracy in edge definition. Methods using fixed external reference systems fail to correct for cardiac movement, and methods which do attempt to correct for this may introduce errors of their own. Thus, current techniques are hardly ideal. They are even less well suited for functional assessment of aneurysmal regions.

The nuclear determination of ejection fraction depends only on count rate changes within a region of interest and makes no assumptions about the shape of the ventricle. It is a simple matter to assign a region of interest to the left ventricular phase image which excludes any region of high phase (and therefore a possible aneurysm) and thereby determine contractile segment ejection fraction. This variable, which has been shown to be the strongest predictor of survival 


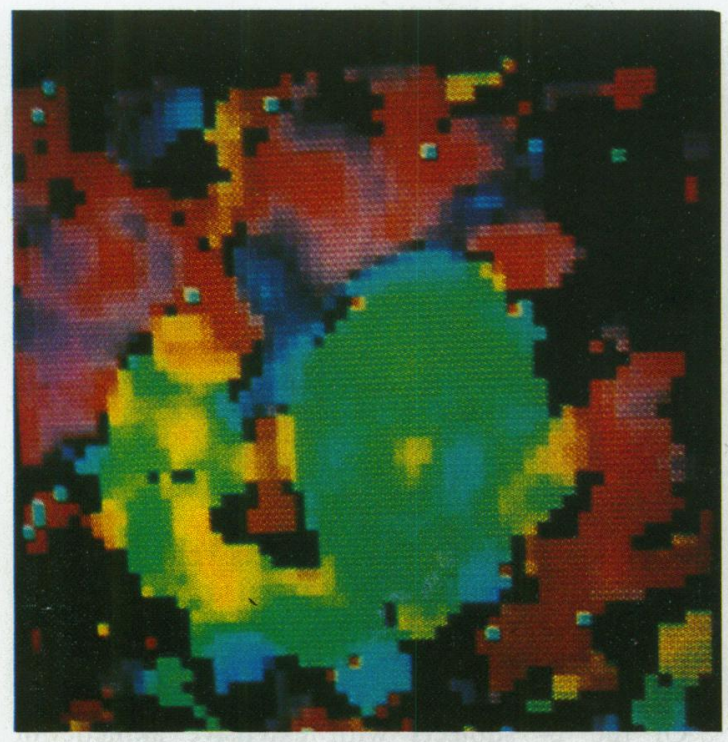

Fig. 3

after aneurysmectomy, ${ }^{7-10}$ can therefore be determined without any of the geometrical assumptions inherent in previous methods. ${ }^{7-13}$ Because there is a continuous distribution of phase a value has to be chosen above which display elements are excluded as aneurysm and below which they are included as contractile segment. Fig. 3 and 4 illustrate the problem. In both there is an area of high phase representing an aneurysm. In Fig. 3 the remaining ventricle is low phase and obviously represents the contractile segment. In Fig. 4 the low phase region is small and there is a large area of intermediate phase. For determination of contractile segment ejection fraction we arbitrarily chose $240^{\circ}$ as the distinction between aneurysm and contractile segment as this was simple to detect on the phase image (red) and only found in those subjects with radiographic extensive akinesis or aneurysm. Using this technique in three subjects there was a good agreement between preoperative contractile segment ejection fraction and postoperative left ventricular ejection fraction. In addition there was a pronounced improvement in the phase images of these subjects which is underestimated by the mean segmental phase values. In each case a region of high phase present preoperatively was not present postoperatively.

Fig. 5 and 6 show volume curves from the contractile and aneurysmal segments of Fig. 3 and 4, respectively. As tracer concentration must be the same in both regions at equilibrium, the ratio of their peak activities reflects the ratio of their volumes. Thus, we cannot only predict postoperative left

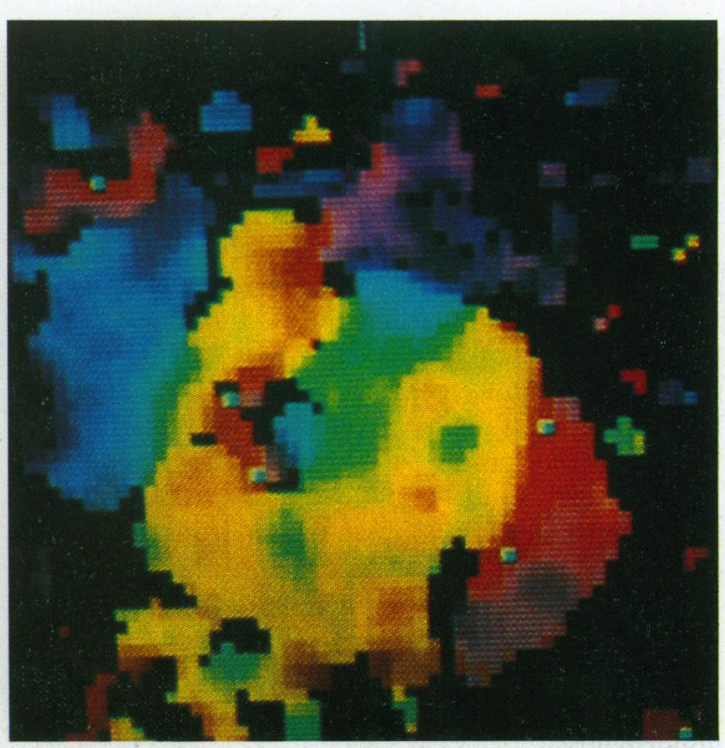

Fig. 4 .

ventricular ejection fraction but we can also estimate the volume of the aneurysm. Thus, subjects with aneurysms on the phase image fall into three groups, those with large aneurysms and low contractile segment ejection fractions $(37,40,46,60)$, those with

\section{CS}

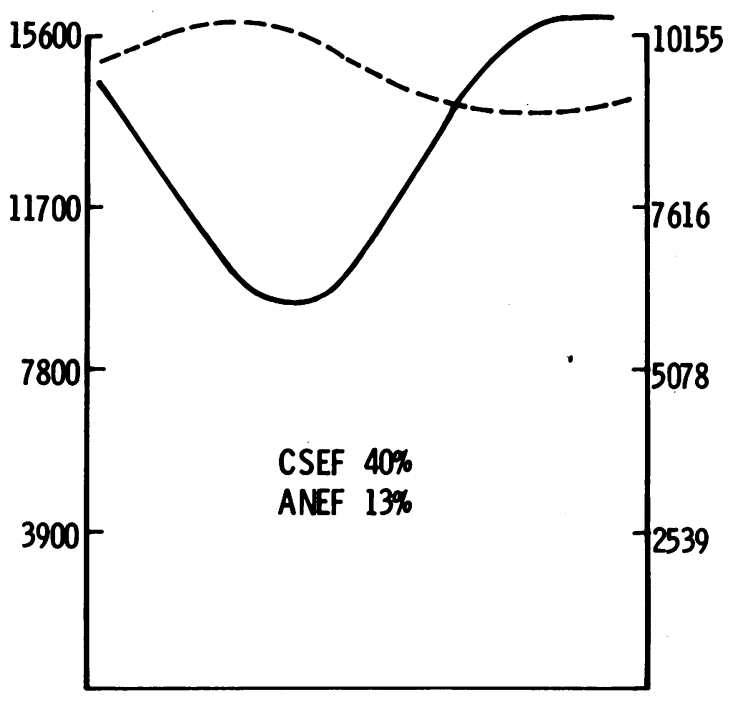

Fig. 5 Volume curves from contractile segment (CS) and aneurysm $(A N)$ of the patient whose phase image is shown in Fig. 3. CSEF, contractile segment ejection fraction; $A N E F$, aneurysm emptying fraction. 


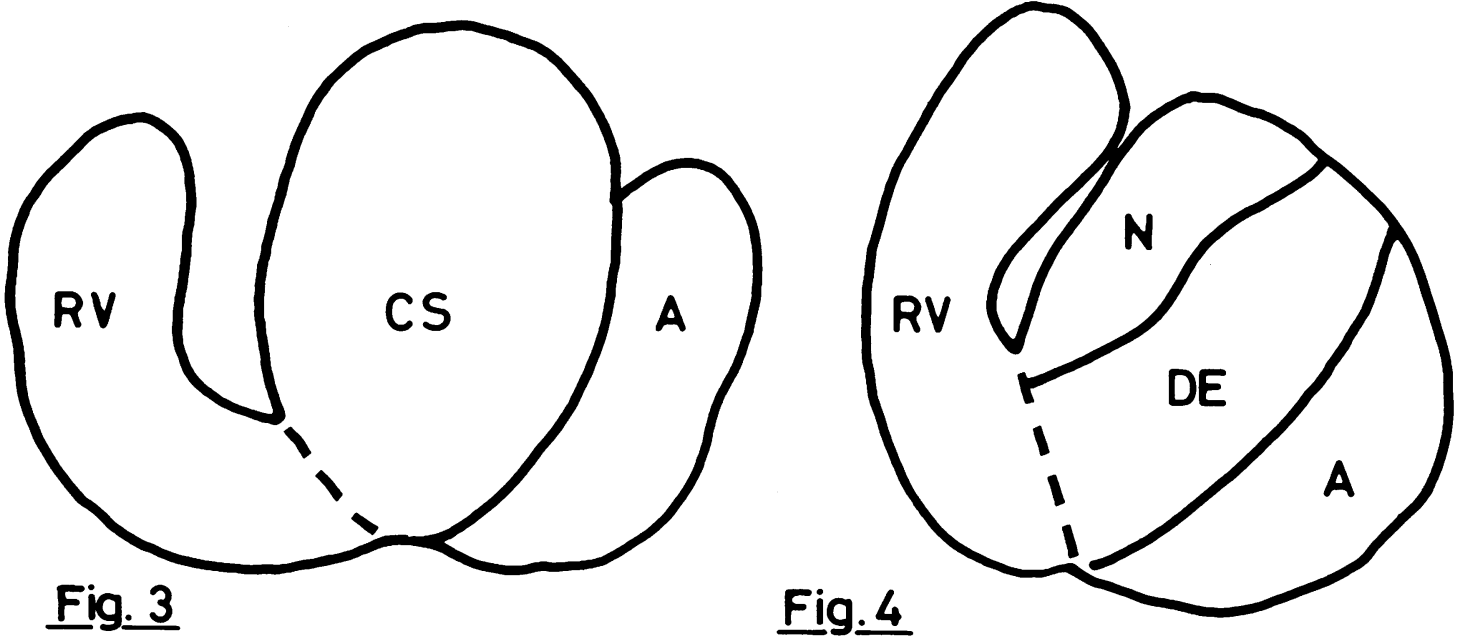

Fig. 3 Phase image showing a left ventricular aneurysm and relatively normal emptying of the rest of the left ventricle. $R V$, right ventricle; $A$, aneurysm; $C S$, normally emptying contractile segment of the left ventricle.

Fig. 4 Phase image showing a left ventricular aneurysm with delayed emptying of much of the contractile segment. $R V$, right ventricle; $A$, aneurysm; $D E$, region of the left ventricle with delayed emptying; $N$, normally emptying portion of the left ventricle.

CS

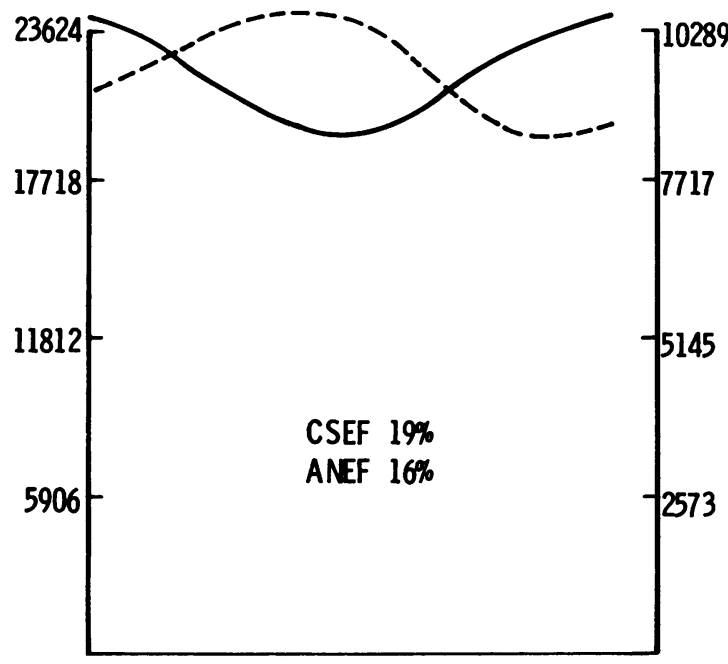

Fig. 6 Volume curves from the contractile segment.(CS) and aneurysm $(A N)$ of the patient whose phase image is shown in Fig. 4. CSEF, contractile segment ejection fraction; $A N E F$, aneurysm emptying fraction.

large aneurysms and high contractile segment ejection fractions $(38,39,47,48,50,56,57,63)$, and those with small aneurysms and high contractile segment ejection fractions $(42,58,67,68)$. Operations for heart failure would obviously be most beneficial in the second group and two of the three operative subjects fall into this category. The third had a small aneurysm and was operated upon for control of recurrent ventricular tachycardia. The subject fell into the third category.

Other nuclear techniques have been applied to the detection of ventricular aneurysm, as indeed has phase imaging itself. Soon after the detection of regional wall motion abnormalities from gated blood pool scintigraphy, ${ }^{14}$ the technique was applied to the detection of paradoxical motion. ${ }^{15}$ This approach, however, depends upon the movement of a mathematical derived edge which does not always reflect actual wall movement. The paradox image ${ }^{16}$ shows regions with higher activity at end-systole (as defined from the ventricular activity time curve) than at end-diastole. Thus it can detect regions with paradoxical filling. It gives no information about other ventricular regions even those with delayed emptying of an extreme degree.

The phase image was initially developed by Adam and co-workers. They realised the potential of the technique in the detection of aneurysms ${ }^{17}{ }^{18}$ but so far there have been few reports of clinical results. In a preliminary study ${ }^{19}$ a reasonable agreement was found between abnormal wall motion and phase delay. Others have also used the technique to assess the effect of conduction disorders on contraction patterns. ${ }^{20}$

The phase image is one of a series of parametric images each one representing the spatial distribution of a particular functional variable such as stroke volume or ejection fraction. ${ }^{21} 22 \mathrm{We}$ use, routinely, a 
combination of such images to differentiate between, for example, phase delays caused by conduction disorders (normal stroke volume image) or infarction (reduced regional stroke volume).

\section{CRITICISM OF TECHNIQUE}

The chief worry concerns the use of a fixed external reference system. No correction is made for cardiac movement. Theoretically this would either produce or mask regions of high phase. This is unlikely to be a problem in hypokinetic ventricles with little overall cardiac movement and it does not seem to be a problem in the group 1 subjects who presumably have vigorously contracting ventricles as no spurious region of high phase was found. It may be part of the explanation for some of the discrepancies observed when comparing phase and angiographic results, though, as already mentioned, there are alternative explanations. This factor may also be operative in the surgical patients in whom the pericardium has been opened and who may, therefore, have pronounced cardiac movement. The extent of the improvement in the phase images was so obvious that this was unlikely to have been artefactual.

Another problem derives from the need to use the left anterior oblique projection for equilibrium studies. This leads to foreshortening of the left ventricle and superimposition of the territories of different coronary arteries. In addition, small basal abnormalities may be obscured because of absorption of radiation from these deeper regions by more superficial regions. The problem may well be overcome by extending the technique to the first-pass studies which can be performed in the right anterior oblique projection. Such studies would also be more suitable for a detailed phase/angiogram comparison as the right anterior oblique projection is superior for wall motion asessment.

The reproducibility of our present technique is good, with a mean difference of $6 \%$ between estimated mean segmental phase values in sequential studies. This could probably be further improved by prolongation of data accumulation time, which in this study was relatively short (60 to 90 seconds). This was done because we felt the need to develop a technique that could easily be extended to exercise studies that require high temporal resolution to detect short lived abnormalities. For routine rest studies end-diastolic intraventricular counts could easily be doubled.

On several occasions the use of mean segmental phase values obscured the magnitude of the peak phase delay. This was obvious on inspection of the phase image but, if necessary, the problem could easily be overcome by the use of a greater number of smaller segments.

\section{Conclusion}

Phase imaging represents an important advance in the detection and functional assessment of regional left ventricular disease. The technique is relatively noninvasive and the results are reproducible. Intraventricular regions of high phase are associated with total occlusion of a major coronary artery, low values of ejection fraction, and significant abnormalities of wall motion.

The technique greatly facilitates the estimation of contractile segment ejection fraction in subjects with left ventricular aneurysm and helps predict the results of aneurysmectomy.

This work is supported by a grant from the Sir Jules Thorn Charitable Trust.

\section{References}

1 Bossuyt A, Deconinck F, Lepoidre R, Joncker M. The temporal Fourier transform applied to functional isotope imaging. In: Information processing in medical imaging. (6th International Conference.) Institut National de la Santé et de la Recherche Medicale 1979; 88: 397-408.

2 Durrer D, VanDam RT, Freud GE, Janse MJ, Meijler FL, Arzbaecher RC. Total excitation of the isolated human heart. Circulation 1970; 41: 899-912.

3 Clayton PD, Bulawa WF, Klausner SC, Urie PM, Marshall HW, Warner HR. The characteristic sequence for the onset of contraction in the normal human left ventricle. Circulation 1979; 59: 671-9.

4 Chaitman BR, DeMots H, Bristow JD, Rösch J, Rahimtoola SH. Objective and subjective analysis of left ventricular angiograms. Circulation 1975; 52: 420-5.

5 Leighton RF, Pollack MEM, Welch TG. Abnormal left ventricular wall motion at mid-ejection in patients with coronary heart disease. Circulation 1975; 52: 238-44.

6 Johnson LL, Ellis K, Schmidt D, Weiss MB, Cannon PJ. Volume ejected in early systole. A sensitive index of left ventricular performance in coronary artery disease. Circulation 1975; 52: 378-89.

7 Arthur A, Basta L, Kioschos M. Factors influencing prognosis in left ventricular aneurysmectomy (abstract). Circulation 1972; 45/46, suppl II: 127.

8 Watson LE, Dickhaus DW, Martin RH. Left ventricular aneurysm. Preoperative hemodynamics, chamber volume, and results of aneurysmectomy. Circulation 1975; 52: 868-73.

9 Lee DCS, Johnson RA, Boucher CA, Wexler LF, McEnany MT. Angiographic predictors of survival following left ventricular aneurysmectomy. Circulation 1977; 55/56, suppl II: $12-8$.

10 Gorlin R, Klein MD, Sullivan JM. Prospective correlative study of left ventricular aneurysm. Mechanistic concept and clinical recognition. Am $\mathcal{F}$ Med 1967; 42: 512-31. 
11 Dymond DS, Stephens J, Stone D, et al. Assessment of function of contractile segments in patients with left ventricular aneurysms by quantitative first pass radionuclide ventriculography. $\mathrm{Br}$ Heart $\mathcal{F} 1980 ; 43$ : 125-33.

12 Field BJ, Russell RO Jr, Dowling JT, Rackley CE. Regional left ventricular performance in the year following myocardial infarction. Circulation 1972; 46: 679-89.

13 Sesto M, Schwarz F, Thiedemann KU, Flameng W, Schlepper $M$. Failure of aneurysmectomy to improve left ventricular function $\mathrm{Br}$ Heart $\mathcal{F}$ 1979; 41: 79-88.

14 Zaret BL, Strauss HW, Hurley PJ, Natarjan TK, Pitt B. A non invasive scintiphotographic method for detecting regional ventricular dysfunction in man. $N$ Engl $\mathcal{F} \mathrm{Med}$ 1971; 284: 1165-70.

15 Rigo P, Murray M, Strauss HW, Pitt B. Scintiphotographic evaluation of patients with suspected left ventricular aneurysm. Circulation 1974; 50: 985-91.

16 Holman BL, Wynne J, Idoine J, Zielonka J, Neill J. The paradox image: a noninvasive index of regional leftventricular dyskinesis. $\mathcal{F ~ N u c l ~ M e d ~ 1 9 8 0 ; ~ 2 0 : ~ 1 2 3 7 - 4 2 . ~}$

17 Geffers H, Adam WE, Bitter F, Sigel H, Kampman H. Data processing and functional imaging in radionuclide ventriculography. Information processing in medical imaging. (5th International Conference held Nashville, Tennessee.) Biomedical Computing Technology Information Center, 1977.

18 Adam WE, Tarkowska A, Bitter F, Stauch M, Geffers H. Equilibrium (gated) radionuclide ventriculography. Cardiovasc Radiol 1979; 2: 161-73.

19 Pavel D, Swiryn S, Lam W, Byrom E, Sheikh A, Rosen $\mathrm{K}$. Veniricular phase analysis of radionuclide gated studies (abstract). Am F Cardiol 1980; 45: 398.

20 Links JM, Douglass KH, Wagner HN Jr. Patterns of ventricular emptying by Fourier analysis of gated bloodpool studies. F Nucl Med 1980; 21: 978-82.

21 Bodenheimer MM, Banka VS, Fooshee CM, Gillespie JA, Helfant RH. Detection of coronary hea:t disease using radionuclide determined regional ejection fraction at rest and during handgrip exercise: Correlation with coronary arteriography Circulation 1978; 58: 640-8.

22 Maddox DE, Holman BL, Wynne J, et al. Ejection fraction image: a noninvasive index of regional left ventricular wall motion. Am F Cardiol 1978; 41: 1230-8.

Requests for reprints to Dr S Walton, Department of Cardiology, Middlesex Hospital, Mortimer Street, London W1N 8AA. 